\title{
reviscafuences
}

ISSN: 1575-7072 | e-ISSN: 2172-7775

Páginas: 190-202

Recibido: 2019-10-30

Revisado: 2020-03-23

Aceptado: 2020-04-01

Preprint: 2020-07-15

Publicación Final: 2020-09-15 www.revistascientificas.us.es/index.php/fuentes/index

DOI: https://doi.org/10.12795/revistafuentes.2020.v22.i2.06

\section{La interactividad en la educación a distancia: un instrumento para su diagnóstico}

\author{
Interactivity in distance education: an instrument for diagnosis
}

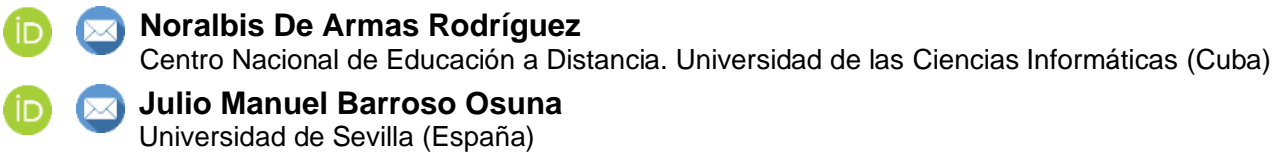

\begin{abstract}
Resumen
Uno de los factores de éxito en el proceso de enseñanza- aprendizaje en la educación a distancia, radica en el desarrollo de la interactividad vista como una variable que influye en la motivación y con ello en el compromiso de los estudiantes hacia el estudio. Pero al mismo tiempo muchos autores plantean que la interactividad es una variable crítica que requiere actualización y mejora. Basado en esto, es importante la realización de un instrumento que permita una retroalimentación constante sobre la interactividad en la educación a distancia. Este artículo tiene como objetivo describir el diseño y validación de un cuestionario para diagnosticar el estado actual de la interactividad en esta modalidad de estudio. La muestra para el diseño del instrumento estuvo compuesta por 253 profesores. Se aplicaron pruebas de validación psicométricas para indagar sobre su fiabilidad y validez. Los resultados manifiestan valores adecuados. Además, se aplicó el análisis factorial exploratorio que arrojó siete factores. La versión final del instrumento incluye un total de siete indicadores y 42 ítems. La aplicación de este instrumento permitirá diagnosticar el estado actual de la interactividad tomando en consideración las interacciones y sobre esta base proponer acciones de mejoras. Estos resultados serán un aporte en el campo de la tecnología educativa, particularmente en el área de la educación a distancia.
\end{abstract}

\begin{abstract}
One of the success factors in the teaching-learning process in distance education lies in the development of interactivity seen as a variable that influences motivation and with it the commitment of students to study. But at the same time, many authors argue that interactivity is a variable criticism that requires updating and improvement. Based on this, it is important to create an instrument that allows constant feedback on interactivity in distance education. This article aims to describe the design and validation of a questionnaire to diagnose the current state of interactivity in this study modality. The sample for the design of the instrument was made up of 253 teachers. Psychometric validation tests were applied to inquire about their validation and validity. The results manifest specific values. In addition, exploratory factor analysis was applied, which yielded seven factors. The final version of the instrument includes a total of seven indicators and 42 items. The application of this enabled instrument diagnoses the current state of interactivity taking into account the interactions and on this basis propose improvement actions. These results will be a contribution in the field of educational technology, particularly in the area of distance education.
\end{abstract}

\section{Palabras clave / Keywords}

educación a distancia, interacción, instrumento, cuestionario, diseño, experto. distance education, interaction, instrument, diagnosis, design, expert. 


\section{Introducción}

En la educación a distancia $(E a D)$ el proceso de enseñanza aprendizaje (PEA) se caracteriza por la separación del profesor y el estudiante en tiempo y espacio. En este sentido la constante relación de intercambio entre estos actores juega un papel importante (Aretio, 2014; Cabero et al., 2015; Guerra et al., 2020). Lo expresado se relaciona con la Teoría de la Comunicación e Interacción integrada a la Teoría del Diálogo Didáctico Mediado (DDM). La teoría se fundamenta en la comunicación a través de los medios por un lado con los materiales permitiendo el autoestudio y por otro mediante las vías de comunicación potenciando la interactividad vertical y horizontal (García-Aretio, 2016; Vlachopoulos \& Makri, 2019).

La interactividad en la EaD es analizada por numerosos autores como una vía importante para mejorar la calidad educativa, por el crecimiento del número de estudiantes que, por sus características, apuestan por esta modalidad de estudio (Salmon, 2014; Villanova, 2016; Mauri, Onrubia, Coll, y Colomina, 2016; Silva, 2017). Sin embargo, es considerada una variable crítica que exige transformación y mejora para una formación de calidad (Huss, Sela y Eastep, 2015; Kahveci, 2005; Wei et al., 2015;Stone \& Springer, 2019 ).

Para lograr la transformación de la interactividad en la EaD, es importante el desarrollo de investigaciones (Fainholc, 2004; García-Aretio, 2019; García-Pérez y García-Aretio, 2014; Williamson, Potter \& Eynon, 2019) para la creación de entornos comunicativos que potencien la individualización y desarrollen la colaboración entre los participantes en un contexto mediado tecnológicamente y sin coincidencia física y espacial.

Lo anterior supone, conocer la percepción de los profesores sobre el estado real de la interactividad en su contexto. La elaboración y aplicación de un instrumento puede ser útil. Con los resultados obtendremos información significativa para realizar proyecciones y estrategias en aras de contribuir a orientar el uso adecuado de la interactividad a favor de la calidad del PEA en esta modalidad de estudio.

En consecuencia, el objetivo de este artículo es describir el proceso de diseño y validación de un cuestionario "ad hoc" para diagnosticar el estado actual de la interactividad en la educación a distancia, desde la visión de los profesores.

\section{Metodología}

\subsection{Metodología}

Para el diseño del cuestionario se siguieron las fases habituales para la construcción de instrumentos de este tipo, expresadas por Albert-Gómez (2006) y utilizadas por Cabero-Almenara, Fernández-Batanero, \& Córdoba-Pérez (2016). Dichas fases son: revisión de la literatura, elaboración de la primera versión del instrumento y formulación de los ítems, aplicación del instrumento a la técnica del juicio de experto para la evaluación de la validez de contenido, elaboración de la versión final y obtención del grado de fiabilidad del instrumento.

\subsection{Participantes}

Se dividieron en dos grupos: un primer grupo encargado de evaluar el grado de validez del contenido estaba formado por 17 expertos en temas de educación a distancia, seleccionados a partir de un muestreo intencional: 12 de distintas universidades nacionales y 5 de universidades extranjeras. De estos profesores, 14 tenían el grado de doctor y tres con el grado de master. Todos con experiencia docente promedio de 26.8 años y de ellos más de 11 años en la educación distancia dedicados a tiempo completo como docentes, garantizando así actualidad y dominio de la información brindada.

Un segundo grupo, para evaluar el grado de fiabilidad del instrumento seleccionado a partir de un muestreo no probabilístico, intencional, formado por 253 profesores vinculados a carreras a distancia del país. De ellos el $41,5 \%$ corresponden al sexo masculino y el $58,5 \%$, al sexo femenino. Los resultados porcentuales, muestran que existen proporciones cercanas en cuanto a la cantidad de hombres y de mujeres con un leve aumento de la proporción de estas últimas.

\subsection{Procedimientos}

El desarrollo de la metodología para diseñar y validar el cuestionario siguió los procedimientos siguientes:

- Diseño del cuestionario ad hoc, a partir de la revisión de estudios similares. 
- Análisis de la validez del contenido mediante el método Delphi, lo cual permitió tener una versión del cuestionario más acabada teóricamente, tomando en consideración las opiniones de los expertos.

- Análisis dimensional del constructo, mediante un análisis factorial exploratorio (AFE).

- Evaluación del grado de fiabilidad a partir el coeficiente Alfa de Cronbach.

- Obtención de la última versión del cuestionario.

Los resultados para cada caso se exponen en el siguiente apartado.

\section{Resultados}

\subsection{Revisión de la literatura y construcción del cuestionario inicial}

La interactividad es un concepto importante en cualquier modalidad de enseñanza, que ha sido definida e identificada por muchos autores como una variable que influye en la motivación y con ello en el compromiso de los estudiantes hacia el estudio (Kishabale, 2019; Mauri et al., 2016). Por lo tanto, en la EaD para que el Proceso de Enseñanza Aprendizaje sea de calidad, el desarrollo de la interactividad es determinante en función de las estrategias de enseñanza que promuevan y estimulen las diferentes interacciones para el aprendizaje.

Pudiera entonces, realizarse la pregunta: ¿existen instrumentos, indicadores y/o documentos para valorar, evaluar o diagnosticar la interactividad en la EaD?

La idea anterior sirvió para la revisión de la literatura llegando a la conclusión de que existen líneas diferentes de investigación. Sobre esta base se seleccionaron dos de ellas por ser las más cercanas al contenido del trabajo. La primera sobre el estudio de la interactividad de forma directa ya sea de cursos o de materiales a utilizar en la educación a distancia u otra modalidad (Garzón et al., 2017; Huss, Sela, Eastep y Huss, 2015; Zolórzano-García \& Navío-Marco, 2019). Y la segunda línea sobre estudios que incluyen los distintos tipos de interacciones (Garrison y Cleveland-Innes, 2005; Madland y Richards, 2016; Siemens, Gasević, y Dawson, 2015; Wang, Chen, y Anderson, 2014; Er \& Er, 2016), por ser estas el sustento para el desarrollo de la interactividad en la EaD, desde la mirada de este trabajo.

En cuanto a la primera línea dirigida a los trabajos que incluyen interactividad en alguna forma de enseñanza o contextos específicos. Kahveci (2007) diseñó una encuesta para recopilar las percepciones de los profesores acerca de la interactividad educativa. O.M.Oassim-Al-shboul, Sabiote, y Álvarez-Rodríguez (2015) desarrollaron una encuesta ad hoc para determinar las percepciones de los profesores, acerca de la implementación de la educación a distancia en su contexto a través de cuatro dimensiones que valoran de forma implícita la interactividad.

Ruiz-Corbella y Aguilar-Feijoo (2017) diseñaron un cuestionario de autoevaluación sobre competencias del docente universitario para mejorar su práctica en entornos virtuales e incluyeron algunas dimensiones relacionadas con la medición pedagógica, la tutoría y el acompañamiento del estudiante considerados importante para el desarrollo de las interacciones.

También existen otros estudios sobre recursos y materiales didácticos (libros, videos, cursos) que uno de sus indicadores como parte del diseño o para evaluar la calidad, incluyen la interactividad, por ejemplo: Bozkurt y Bozkaya (2015); Morales y García (2015); Ettazarini, (2017); Geri, Winer, y Zaks (2017); Mauri et al. (2016); Guerrero y Madrid (2019); García-Hernández y González-Ramírez (2017); Jiménez-Palmero, Mora-Núñez y Cuadros-Muñoz (2016); García-Hernández \& González-Ramírez (2018).

Por lo que se aprecia, se observa que la interactividad de manera general se evalúa como un atributo dentro de un recurso o medio educativo. De manera particular en la educación a distancia, desde la visión de los autores, aun cuando la interactividad es considerada muy importante resultan más escasos los trabajos que la abordan como un proceso reflexivo a partir de las interacciones que se establecen con el contenido y entre los sujetos.

La segunda línea incluye los distintos tipos de interacciones ya sea sobre la forma de evaluarlas 0 desarrollarlas. Son numerosos los instrumentos y modelos que proponen o evalúan los distintos tipos de interacciones. Por ejemplo, Canós-Rius y Catases (2014), en el cuestionario para el análisis de la interacción estudiante-profesor, lo dividieron en tres dimensiones o bloques: uso de las TIC para la interacción, herramientas utilizadas en la interacción, finalidad real de las herramientas de interacción.

Otros investigadores utilizan el modelo de Garrison y Cleveland-Innes (2005) para el análisis de las interacciones en cursos en línea (Akar, Mardikyan, y Dalgic, 2018; Blayone et al., 2017; Gutiérrez-Santiuste y 
Gallego-Arrufa, 2017; Nolan-grant, 2019; Stewart, 2019; Thongsawat, Davidson-Shivers y Chatphoomiphong, 2019; 2011; Zhu, Herring, y Bonk, 2019).

Siemens, Gasević y Dawson (2015) plantean entre otros elementos, que los cursos en línea deben proporcionar un buen apoyo para las interacciones entre estudiantes y otros estudiantes, entre el estudiante con el tutor o profesor y que el contenido proporcionado debe ser visualmente atractivo e interactivo.

Como resultado, en esta segunda línea sobre las interacciones no ocurre lo mismo que en la primera línea, pues, existen estudios que reflejan vías y formas de cómo evaluarlas y diferentes modelos para desarrollarlas de forma general. Es importante señalar que para esta investigación, las interacciones son primordiales para favorecer la interactividad y contribuir al logro de los objetivos de aprendizaje de forma individual y grupal (De Armas y Barroso, 2018).

La revisión de la literatura reveló que existen investigaciones que incluyen de una forma u otra, elementos asociados a la interactividad y a las interacciones desde diferentes perspectivas. Estos elementos sirvieron de base para determinar las dimensiones del cuestionario. El análisis se puede observar en la tabla 1 y abarcó el título del trabajo, los autores y las dimensiones e indicadores que evalúan o proponen según el objetivo para cada caso.

Esta revisión proporcionó las cuatro dimensiones que podían ser parte de la versión inicial del cuestionario: características de la interactividad, tipos de actividades, tipos de recursos educativos y tipos de medios tecnológicos y/o servicios telemáticos. A partir de ellas, se identificaron 11 indicadores y 90 ítems.

\section{Tabla 1}

Trabajos revisados que incluyen dimensiones e indicadores relacionados con la interactividad

\section{Fuentes relacionadas}

Kahveci (2007)

Canós-Rius y Catases (2014)

-Bozkurt y Bozkaya (2015)

-García-Hernández y González-

Ramírez (2018)

Morales y García (2015)

Huss, Sela, Eastep, y Huss (2015)

Siemens, Gasević y Dawson (2015)

Park (2014)

Ruiz-Corbella y Aguilar-Feijoo, (2017)

Geri et al. (2017)

Ortiz y Ortiz (2019)

\section{Dimensiones}

- Atributos de la interactividad en función de las teorías de motivación y de las teorías aprendizaje.

- Herramientas utilizadas para las interacciones.

- Contenido.

- Interactividad.

- Tecnología.

- Efecto en la instrucción.

- Facilidad de uso.

- Interacción estudiante- estudiante.

- Interacción estudiante-profesor.

- Interacción estudiante contenido.

- Interacciones entre estudiantes y estudiantes

- Aprendizaje cooperativo y colaborativo.

- Interacción del estudiante con el tutor.

- Información sobre el progreso del aprendizaje.

- Contenido visualmente atractivo.

- Calidad del aprendizaje en línea.

- Calidad de los materiales de aprendizaje.

- Calidad de la reflexión y la comunicación en línea.

- Competencias didáctico-metodológicas.

- Competencias de tutoría y acompañamiento.

- Evaluación del aprendizaje y de la actividad docente.

- Integración de elementos interactivos en videos en línea.

- Aprendizaje colaborativo.

- Construcción del conocimiento.

- Interacción con los compañeros.

- Aprendizaje colaborativo. 
Guerrero y Madrid (2019)

- Claridad en las actividades.

- Interacción estudiante-profesor.

- Formato de los contenidos educativos.

- Retroalimentación a las actividades.

La versión inicial estuvo formada por dos partes: una dirigida a datos sociodemográficos de los participantes: sexo, edad, categoría docente, años en la educación a distancia y la otra dirigida a la recogida de información propia de la temática del cuestionario, con una escala Likert y cinco grados de respuestas.

Es importante resaltar que el cuestionario diseñado es específico para evaluar o diagnosticar el desarrollo de la interactividad en la educación a distancia, desde la visión de los profesores, esto sin duda lo hace diferente del resto de los instrumentos analizados y resulta de un gran valor para las prácticas educativas en la EaD.

\subsection{Evaluar el grado de validez de contenido del instrumento}

Para evaluar el grado de validez, se tuvo en cuenta el criterio de Escobar-Pérez y Cuervo-Martínez (2008). Se utilizó el juicio de expertos mediante la técnica Delphi (Barroso \& Cabero, 2013; Aguilar-Gavira y Barroso). La organización de la misma siguió tres etapas propuestas por Blasco y López (2010): preliminar, exploratoria y final.

La etapa preliminar dirigida a la selección de los expertos que validaron el instrumento tuvo en cuenta criterios como: formación con grado académico de máster y/o doctor, años de experiencia en la educación a distancia y su aceptación para colaborar. Del total de 25 profesores de universidades cubana y extranjeras, respondieron 17.

Para garantizar la calidad se utilizó la combinación del biograma con la información obtenida del índice de competencia experta, en este caso superior a 0,8 puntos por lo que el $100 \%$ de los encuestados tenían un nivel de competencia alto. Los 17 fueron seleccionados como expertos en EaD.

La etapa exploratoria dirigida al intercambio de información con los expertos, en dos rondas. En la primera se envió por correo electrónico dos documentos: uno con el cuestionario inicial y el otro para la valoración del contenido, el experto debía expresar su valoración general de la estructura y contenido, desde lo cuantitativo y cualitativo.

La segunda ronda, tomó en consideración las propuestas y sugerencias derivadas de la primera ronda, se rediseñó la segunda versión del cuestionario y se les envió a los expertos para su valoración, junto al resultado del análisis estadístico.

La etapa final dirigida a la construcción de la versión inicial del cuestionario. Sobre la estructura y contenido general del cuestionario desde el punto de cuantitativo, los expertos recomendaron que cinco indicadores ubicados en la categoría de adecuado debían ser analizados para su modificación o eliminación, el resto fueron considerados para ser parte del instrumento.

Desde el punto de vista cualitativo algunos indicadores se sugirieron ser analizados para su modificación o eliminación. Las insuficiencias de estos indicadores estuvieron en la coherencia en la estructura del instrumento, en la claridad de la redacción de los ítems, en la secuencia y agrupación de los ítems para facilitar la comprensión.

Para los resultados generales cualitativo y cuantitativo, se revisaron los criterios de los expertos sobre cada uno de los ítems para ver en qué medida deben mantenerse, eliminarse, modificarse o añadir algunos. Se utilizaron los estadígrafos descriptivos: la media, la desviación típica y la distribución de frecuencias.

Se analizaron los 11 indicadores y el banco de 90 ítems, la gran mayoría de los ítems sus puntuaciones medias oscilaban entre 1.00 y 1.17 o sea a favor de mantenerlos expresado por un $53 \%$ de los expertos, no obstante, se observó que entre un $42 \%$ y $47 \%$ coincidieron en que los 14 ítems del indicador "interacción estudiante - profesor", y los nueve del indicador "interacción estudiante - estudiante" debían fusionarse o sea modificarse.

Los ítems del indicador "tipos de recursos educativos" donde un $42 \%$ coincidieron en que deben fusionarse con algunos de los ítems del indicador "tipos de medios tecnológicos". De igual modo los 15 ítems unidos al indicador "tipos de medios tecnológicos", deben eliminarse pues entre un $63 \%$ y un $67 \%$ de los expertos así lo reflejan. El resto de los ítems alcanzó un nivel elevado de coincidencia a favor de mantenerse.

Como resultado se eliminaron 15 ítems para un $16,6 \%$ y se fusionaron o modificaron 34 para un $37 \%$, quedando la propuesta en 75 ítems. Se elaboró y se envió la versión nueva a los expertos en una segunda ronda para su validación siguiendo el procedimiento anterior. Los expertos coincidieron en mantener todos los ítems. 
Finalmente, se elaboró el instrumento integrado por 10 indicadores y 75 ítems, cada uno de ellos con una escala tipo Likert de 5 opciones de respuesta. La primera parte está formada por datos sociodemográficos y la segunda por los indicadores e ítems para cada caso.

\subsection{Análisis factorial exploratorio. Validez de constructo}

Después de evaluado el grado de validez mediante juicio de expertos, con la técnica Delphi, se analizó la validez de constructo del instrumento. Para ello, se aplicó el instrumento a 253 profesores y sobre esta base se realizó un análisis factorial exploratorio (AFE).

Inicialmente, para comprobar si es posible realizar el AFE se efectuaron las pruebas de Bartlett y la de KaiserMeyer-Olkin(KMO). Se observa en la tabla 2, que la bondad de ajuste es aceptable, dado que el KMO toma un valor 0.853, superior a 0.6 (Kaiser, 1974) y la prueba de esfericidad de Bartlett muestra una significancia menor a .05 lo que evidencia que la matriz de correlación es distinta a la matriz de identidad.

\section{Tabla 2}

Prueba de KMO y Bartlett para el AFE

Medida de adecuación muestral de KMO

.853

Chi-cuadrado aproximado

8172.905

Prueba de esfericidad de

Bartlett

gl

.703

Sig.

.000

Los resultados indican la idoneidad de la aplicación del AFE para lo cual se utilizó el método de los componentes principales con rotación Varimax. La extracción de los factores a través de la agrupación de los 10 indicadores o componentes originales, desde una primera mirada, produjo siete factores o componentes como autovalores mayores que 1 , o sea tres menos de la composición original, hubo reducción de factores. Para mayor información se hizo necesario, observar el gráfico de sedimentación (Figura 1) lo cual reforzó la idea de proponer una agrupación diferente de ítems, a la primera versión del cuestionario mostrando un fuerte cambio de pendiente a partir del séptimo componente.

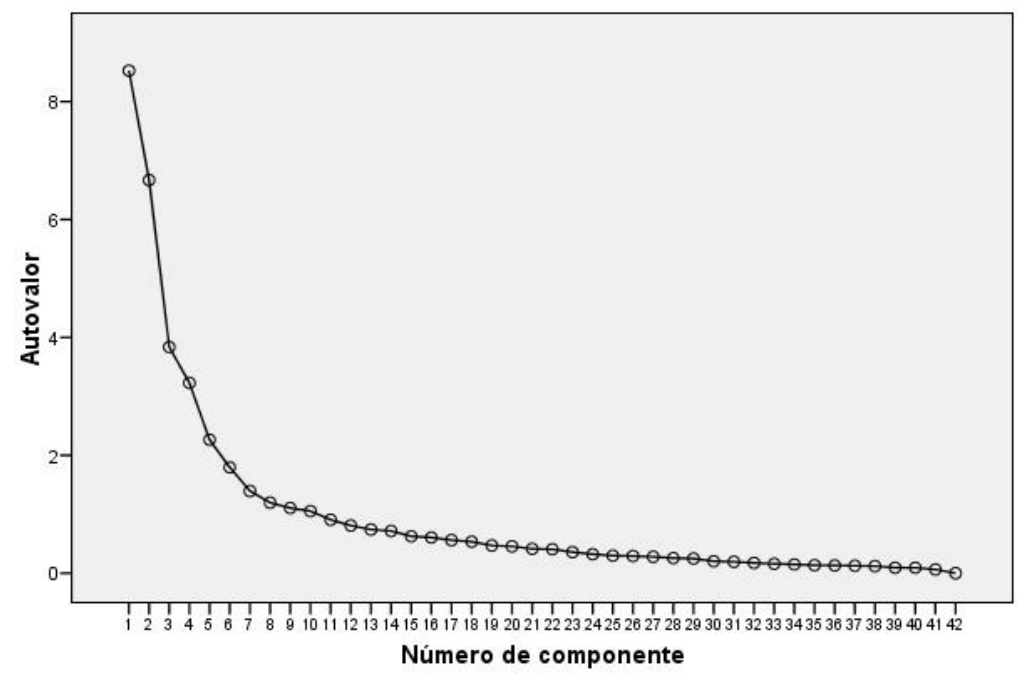

Figura 1 Gráfico de sedimentación 
Al mismo tiempo la tabla 3 sobre la varianza total explicada también declara los siete componentes principales que tienen valores propios mayores que 1 , con una varianza total del $72.74 \%$. Esto quiere decir que estos componentes representan $72.74 \%$ de la información original.

Tabla 3

Método de Kaiser para el AFE

\begin{tabular}{|c|c|c|c|c|c|c|}
\hline \multirow{2}{*}{ Componentes } & \multicolumn{3}{|c|}{ Autovalores iniciales } & \multicolumn{3}{|c|}{$\begin{array}{l}\text { Sumas de las saturaciones al cuadrado de } \\
\text { la rotación }\end{array}$} \\
\hline & Total & $\%$ de varianza & $\%$ acumulado & Total & $\%$ de varianza & $\%$ acumulado \\
\hline 1 & 8.65 & 22.77 & 22.77 & 6.99 & 21.39 & 21.39 \\
\hline 2 & 7.16 & 18.84 & 41.61 & 4.71 & 14.39 & 35.78 \\
\hline 3 & 3.54 & 9.32 & 50.93 & 3.16 & 8.55 & 44.33 \\
\hline 4 & 3.16 & 8.33 & 59.26 & 2.92 & 7.69 & 52.02 \\
\hline 5 & 2.69 & 7.09 & 66.35 & 2.90 & 7.65 & 59.67 \\
\hline 6 & 1.29 & 3.40 & 69.75 & 2.89 & 7.62 & 67.29 \\
\hline 7 & 1.13 & 2.99 & 72.74 & 2.06 & 5.45 & 72.74 \\
\hline
\end{tabular}

La tabla 4 representa la matriz de componentes rotados de cargas factoriales, en este caso se muestran solo los componentes que su carga factorial estuvo por encima de 0.40 , teniendo en cuenta que el número de la muestra es menor que 300 y que puede ser considerado ese valor factorial (Lloret-segura, Ferreres-traver, Hernández-Baeza, y Tomás-Marco, 2014). Bajo este supuesto se redujo la cantidad a 42 ítems y hubo una agrupación diferente de indicadores a la original, a siete componentes, algo que contribuyó al logro de la optimización del instrumento, siendo esto la esencia del AFE (Hefetz y Liberman, 2017).

\section{Tabla 4}

Matriz componentes rotados de cargas factoriales mayor que .04

\begin{tabular}{|c|c|c|c|c|c|c|c|c|c|}
\hline İtems & 1 & 2 & 3 & Ítems & 3 & 4 & 5 & 6 & 7 \\
\hline 1. & .983 & & & 22 & .473 & & & & \\
\hline 2. & .663 & & & 23 & .866 & & & & \\
\hline 3. & .904 & & & 24 & .504 & & & & \\
\hline 4. & 801 & & & 25 & & .528 & & & \\
\hline 5. & .548 & & & 26 & & .677 & & & \\
\hline 6. & .822 & & & 27 & & .784 & & & \\
\hline 7. & .478 & & & 28 & & & .639 & & \\
\hline 8. & .453 & & & 29 & & & .721 & & \\
\hline 9. & .571 & & & 30 & & & .794 & & \\
\hline 10. & .868 & & & 31 & & & .584 & & \\
\hline 11. & .796 & & & 32 & & & & .596 & \\
\hline 12. & & .858 & & 33 & & & & .439 & \\
\hline 13. & & .808 & & 34 & & & & .668 & \\
\hline 14. & & .686 & & 35 & & & & .428 & \\
\hline 15. & & .789 & & 36 & & & & .668 & \\
\hline 16. & & .656 & & 37 & & & & .708 & \\
\hline
\end{tabular}




\begin{tabular}{|c|c|c|c|}
\hline 17. & .429 & 38 & .813 \\
\hline 18. & .557 & 39 & .532 \\
\hline 19. & .814 & 40 & .555 \\
\hline 20. & .505 & 41 & .759 \\
\hline 21. & & 42 & .659 \\
\hline
\end{tabular}

La nueva agrupación teórica, de los siete indicadores con sus ítems puede definirse como sigue: 1) interacción entre los sujetos: estudiante-profesor, estudiante-estudiante, con ítems del 1 al 11 ;2) Interacción estudiantecontenido, con ítems del 12 al 20;3) actividades conceptuales, con ítems del 21 al 24; 4) actividades procedimentales, con ítems del 25 al 27; 5) actividades actitudinales, con ítems del 28 al 31; 6) recursos educativos informáticos, con ítems del 32 al $38 ; 7$ ) medios tecnológicos y servicios telemáticos para promover las interacciones en la educación a distancia con ítems del 39 al 42.

El resultado de la nueva extracción de componentes evidenció que la agrupación de los ítems resultaba más acorde y simplificada, pasando de los 75 ítems originales a 42.

\subsection{Análisis de fiabilidad}

Esta fase permitió determinar el grado de la fiabilidad, vista esta como la consistencia o estabilidad de las medidas de un instrumento cuando el proceso de medición se repite (Cronbach \& Meehl, 1955; Prieto y Delgado, 2010).

El cuestionario fue aplicado a 253 profesores y se utilizó el estadístico descriptivo, Alfa de Cronbach. El valor total de Alfa en la escala de .838 (Tabla 5) indica una correlación muy alta, teniendo en cuenta que correlaciones situadas entre el intervalo 0.8 y 1 se pueden considerar como "muy altas" Esto indica una estabilidad en las respuestas adecuada, por lo que la escala presenta indicios de garantía de calidad.

Tabla 5

Estadísticas de fiabilidad del instrumento

Alfa de Cronbach Número de elementos

Para analizar la relación de cada uno de los ítems, con el total del coeficiente interno alfa alcanzado, se obtuvo la correlación ítem total del instrumento para conocer si la eliminación de algunos de los ítems aumentaría el índice de fiabilidad del instrumento.

Tabla 6

Alfa de Cronbach si se elimina el elemento

\begin{tabular}{cccc}
\hline № & $\begin{array}{c}\text { Alfa de Cronbach si se elimina el } \\
\text { elemento }\end{array}$ & № & Alfa de Cronbach si se elimina el elemento \\
\hline 1. & .829 & 22. & .822 \\
2. & .832 & 23. & .824 \\
3. & .828 & 24. & .834 \\
4. & .830 & 25. & .824 \\
5. & .825 & 26. & .825 \\
6. & .826 & 27. & .830 \\
7. & .838 & 28. & .824 \\
8. & .826 & 29. & .821 \\
9. & .828 & 30. & .822 \\
10. & .825 & 31. & .817 \\
11. & .829 & 32. & .828 \\
12. & .822 & 33. & .828 \\
\hline
\end{tabular}




\begin{tabular}{llll}
\hline 13. & .822 & 34. & .825 \\
14. & .827 & 35. & .828 \\
15. & .827 & 36. & .832 \\
16. & .831 & 37. & .823 \\
17. & .823 & 38. & .829 \\
18. & .828 & 39. & .835 \\
19. & .830 & 40. & .825 \\
20. & .830 & 41. & .826 \\
21. & .824 & 42. & .833 \\
\hline
\end{tabular}

En la tabla 6 se presentaron los resultados alcanzados observándose que no existe ningún valor superior a .838, por lo que eliminar algún ítem no aumentaría la fiabilidad del instrumento.

\section{Discusión}

La interactividad constituye uno de los principales elementos para elevar la calidad de la educación a distancia, sin embargo, en la actualidad, la misma está llamada a un proceso de transformación y mejora expresado por autores como: Cabero (2008); Autor (2018); García - Aretio (2014), (2017), (2019); García Pérez, y García- Aretio (2016).

Se observó en la revisión de la literatura que si bien, no existe uniformidad sobre la definición y evaluación del constructo, se muestra la existencia de diferentes instrumentos que incluyen elementos referidos a la interactividad y a la interacción en diferentes contextos (Kahveci, 2007; Bozkurt y Bozkaya, 2015; Huss, Sela, Eastep, y Huss, 2015). Además de que existen muchos trabajos que resaltan la necesidad desarrollar la interactividad en entornos donde no hay coexistencia de espacio y tiempo.

Se hizo necesario diseñar y validar un instrumento capaz de diagnosticar la interactividad en la EaD. Es determinante tener en cuenta la visión del profesor, por ser ellos los protagonistas para desarrollar la interactividad en su práctica educativa. La construcción del cuestionario tuvo en cuenta elementos de diferentes instrumentos validados reflejados en la tabla 1. Se elaboró el cuestionario inicial, que después de la evaluación de los expertos mediante la técnica Delphi, permitió elaborar una versión más actualizada, estructurado por 10 indicadores y 75 ítems.

Atendiendo a los objetivos propuestos se procedió al estudio de la fiabilidad del instrumento a fin de determinar su idoneidad. El resultado del análisis de consistencia interna fue de .838 . Se realizó un análisis factorial exploratorio lo que facilitó una visión más clara tomando como referencia que la finalidad del AFE, es realizar un estudio preliminar del constructo teórico con mayor precisión, a partir de la mejor agrupación de ítems, así como plantean Lloret-segura et al. (2014) y Hefetz y Liberman (2017). El resultado fue de siete indicadores y 42 ítems, algo importante, desde la visión de los investigadores. Los indicadores se relacionan con :1) interacción entre los sujetos; 2) Interacción estudiante-contenido; 3) actividades conceptuales; 4) actividades procedimentales; 5) actividades actitudinales; 6 ) recursos educativos informáticos; 7) medios tecnológicos y servicios telemáticos para promover las interacciones en la EaD.

Los siete indicadores del estudio, coinciden con las posiciones teóricas de autores como: Cabero y Llorente (2007); Croxton (2014); García-Aretio (2014), (2019); Gutiérrez-Santiuste y Gallego-Arrufat (2017); Mauri et al., (2016); Moore (1989) y Pedrosa y García-Cueto (2014) que plantean que en un entorno a distancia, la interactividad es vital. La misma se sustenta en tres componentes fundamentales: en la preparación de recursos, materiales y contenidos bien elaborados; en las gestiones tutoriales a través de los medios tecnológicos, que tienen como centro la motivación y el fortalecimiento de la autonomía y en el trabajo colaborativo entre los actores del proceso de enseñanza aprendizaje que permiten fortalecer las interacciones sociales.

Existen dos indicadores que centran la atención y que se corresponden con la organización inicial del cuestionario: la interacción entre los sujetos (interacción del estudiante con sus compañeros; interacción del estudiante con su profesor) y el otro indicador es la interacción del estudiante con el contenido. Siendo este último el punto de partida para desarrollar las interacciones entre los sujetos y por consecuencia el desarrollo de la interactividad en la educación a distancia, desde la postura de esta investigación.

Los otros indicadores identificados y agrupados resultaron del estudio teórico de trabajos e instrumentos (Cisneros, Maribel, Chimbo, y Trejo, 2018; Ettazarini, 2017; Mauri et al., 2016) que coinciden en que en un entorno a distancia debe haber presencia de medios tecnológicos tales como: dispositivos móviles, plataformas virtuales, computadoras personales, entre otros. Además, se debe incluir tipos de actividades de 
aprendizaje (Mauri et al., 2016; Silva, 2017) y por consiguiente el tipo de recurso o material educativo que permitirá realizar los tipos de actividades (Morales y García, 2015; Ruiz-Corbella y Aguilar-Feijoo, 2017).

\section{Conclusiones}

La interactividad en la EaD es analizada por numerosos autores como una vía importante para mejorar la calidad educativa en cualquier modalidad de estudio, esta idea es asumida también por los autores de este trabajo.

El estudio realizado, develó que muchas investigaciones incluyen elementos para evaluar la interactividad de forma general siendo insuficiente, desde la visión de los autores de este trabajo, la existencia de un instrumento específico para evaluar o diagnosticar el desarrollo de la interactividad en la educación a distancia, desde la visión de los profesores. En este sentido, es el aporte más significativo de este artículo.

El análisis e identificación de las variables y los indicadores, a partir de la revisión bibliográfica, permitió diseñar el cuestionario para diagnosticar el desarrollo de la interactividad en la educación a distancia desde la visión de los profesores.

Como elemento distintivo de este instrumento, se destaca que el sustento teórico de la variable interactividad en la educación a distancia, sienta su base en un proceso de reflexión coincidiendo con Garrison y ClevelandInnes (2005) en que los estudiantes son capaces de construir significado mediante la reflexión y análisis de las actividades de aprendizajes y los recursos disponibles para solucionar dichas actividades.

Se concluye que este instrumento es válido y fiable para diagnosticar la interactividad en la educación a distancia desde la percepción de los profesores.

\section{Limitaciones y prospectiva}

Para evaluar la dimensionalidad del instrumento, se realizó solo el análisis factorial exploratorio. Se recomienda para investigaciones futuras el análisis factorial confirmatorio pues esclarecerá o corroborará el resultado del AFE.

El instrumento está concebido para diagnosticar la interactividad en la EaD, solo desde la visión de los profesores. Se propone el diseño de otros instrumentos con características similares, desde la visión del estudiante y de directivos docentes.

Describir el proceso de diseño y validación del cuestionario servirá de referencia a otros investigadores. Este puede ser aplicado en cualquier modalidad de estudio para diagnosticar la interactividad en la educación, desde la percepción del profesor, lo cual representa una oportunidad para que las instituciones y los profesores puedan autoevaluar su práctica educativa en la educación a distancia, esta óptica es coincidente con el instrumento de Ruiz-Corbella y Aguilar-Feijoo (2017).

\section{Referencias}

Aguilar-Gavira, S. y Barroso J. (2018). Evaluación de un entorno de formación para la adquisición de competencias tecnológicas en el profesorado universitario. Profesorado. Revista de Currículum y Formación de Profesorado, 22(3), 359-374. https://doi.org/10.30827/profesorado.v22i3.8006

Akar, E., Mardikyan, S., \& Dalgic, T. (2018). User Roles in Online Communities and Their Moderating Effect on Online Community Usage Intention: An Integrated Approach. International Journal of Human-Computer Interaction, 35(6), 495509. https://doi.org/10.1080/10447318.2018.1465325

Albert-Gómez, M. J. (2006). La investigación educativa. Claves teóricas. (McGraw-Hill, Ed.) (McGraw-Hil). Madrid. Barroso, J., \& Cabero, J. (2013). La utilización del juicio de experto para la evaluación de TIC: El coeficiente de competencia experta. Sociedad Española de Pedagogía, 65. https://doi.org/10.13042/brp.2013.65202

Blasco, J. E., \& López, A. M. (2010). Validación mediante método Delphi de un cuestionario para conocer las experiencias e interés hacia las actividades acuáticas con especial atención al windsurf. Ágora para la Educación Física y el Deporte, 12(1), 75-94. https://bit.ly/2JksuCb

Bozkurt, A., \& Bozkaya, M. (2015). Evaluation Criteria for Interactive E-Books for Open and Distance Learning. The International Review of Research in Open and Distributed Learning, 16(5). https://doi.org/10.19173/irrodl.v16i5.2218 Cabero-Almenara, J., Barroso, J., LLorente, M. del C., Díaz, I. M., Marín, O. C. C. G. V., Puente, A. P., ... Sardi, E. E. N. P. M. R. F. M. C. F. (2015). Mitos, prejuicios y realidades de la educación a distancia . Venezuela: Universidad Metropolitana, Caracas. Recuperado a partir de http://libros.uat.edu.mx/omp/index.php/editorialuat/catalog/book/173 Cabero-Almenara, J., Fernández-Batanero, J. M., \& Córdoba-Pérez, M. (2016). Conocimiento de las TIC aplicadas a las personas con. magis, Revista Internacional de Investigación en Educación, 8(17), 157-176. https://dx.doi. org/10.11144/Javeriana.m8-17.ctap 
Canós-Rius, N., \& Catases, M. G. (2014). Uso de las TIC en la interacción profesor- alumno: un estudio de caso en una Escuela de Arte y Superior de Diseño. RELATEC -Revista Latinoamericana de Tecnología Educativa, 13, 63-70. https://bit.ly/3652Zi5

Cronbach, L. J., \& Meehl, P. E. (1955). Construct validity in psychological tests. Psychological Bulletin, 52(4), $281-302$. https://doi.org 10.1037/h0040957

Er, N. F., \& Er, M. (2016). The effects of student-content interaction on academic performance in distance-learning courses. International Journal on New Trends in Education \& their Implications (IJONTE), 7(3), 60-68.

https://doi.org/10.5353/th b3121089

Escobar-Pérez, J., \& Cuervo-Martínez, Á. (2008). Validez de contenido y juicio de expertos: una aproximación a su utilización. Avances en Medición, 6(1), 27-36. https://bit.ly/31Q4Gwm

Ettazarini, S. (2017). Analysis of interactivity and autonomy of existing digital educational resources: the case of Life and Earth Sciences in Morocco. Open Learning: The Journal of Open, Distance and e-Learning, 32(2), 103-117.

https://doi.org/10.1080/02680513.2017.1305884

García-Aretio, L. (2016). El juego y otros principios pedagógicos. Supervivencia en la educación a distancia y virtual. RIED. Revista Iberoamericana de Educación a Distancia, 19, 9-23. https://doi.org/10.5944/ried.19.2.16175

García-Aretio, L. (2017). Educación a distancia y virtual: calidad, disrupción, aprendizajes adaptativo y móvil. RIED. Revista Iberoamericana de Educación a Distancia, 20(2), 9. https://doi.org/10.5944/ried.20.2.18737

García-Aretio, L. (2019). El problema del abandono en estudios a distancia. Respuestas desde el Diálogo Didáctico Mediado. RIED, Revista Iberoamericana de Educación a Distancia, 22(1), 245. https://doi.org/10.5944/ried.22.1.22433 García-Hérnandez, A \& Gonzaléz-Ramírez, T. (2017). Design and assessment of the impact of an e-textbook in the engagement towards the learning of Discrete Mathematics. En Proceedings of 5th International Conference Technological Ecosystems for Enhancing Multiculturality, Spain, (TEEM'17). https://doi.org/10.1145/3144826.3145443 García-Hernández, A., \& González-Ramírez, T. (2018, October). Construction and validation of a questionnaire to assess student satisfaction with mathematics learning materials. En Proceedings of the Sixth International Conference on Technological Ecosystems for Enhancing Multiculturality (pp. 134-138). ACM. https://doi.org/10.1145/3284179.3284204

García-Pérez, M., \& García-Aretio, L. (2014). Líneas de investigación y tendencias de la educación a distancia en américa latina a través de las tesis doctorales. RIED. Revista Iberoamericana de Educación a Distancia, 17(1). https://doi.10.5944/ried.17.1.11581

Garrison, D. R., \& Cleveland-Innes, M. (2005). Facilitating Cognitive Presence in Online Learning: Interaction Is Not Enough. American Journal of Distance Education, 19(3), 133-148. https://doi.org/0.1207/s15389286ajde19032 Blayone, T. J. B., Mykhailenko, O., vanOostveen, R., Grebeshkov, O., Hrebeshkova, O., \& Vostryakov, O. (2017). Surveying digital competencies of university students and professors in Ukraine for fully online collaborative learning. Technology, Pedagogy and Education, 27(3), 279-296. https://doi.org/10.1080/1475939X.2017.1391871 Geri, N., Winer, A., \& Zaks, B. (2017). A learning analytics approach for evaluating the impact of interactivity in online video lectures on the attention span of students. Interdisciplinary Journal of e-Skills and Lifelong Learning, 13, 215-228. https://doi.org/10.28945/3875

Guerra, K., Góme, N. A., Peña, R., Pintado, N., Fuster-Guillén, D., \& Palacios-Garay, J. (2020). Prácticas educativas inclusivas a través de la educación a distancia. Experiencias en Cuba. Propósitos y Representaciones, 8. https:// dx.doi.org/10.20511/pyr2020.v8n2.446

Guerrero, K. F., \& de la. Madrid, M. C. L. (2019). Evaluación de cursos en línea desde la perspectiva del estudiante: un análisis de métodos mixtos. Perspectiva Educacional, 58(1), 92-114. https://doi.org/ 10.4151/07189729

Gutiérrez-Santiuste, E., \& Gallego-Arrufat, M.-J. (2016). Type and degree of co-occurrence of the educational communication in a community of inquiry. Interactive Learning Environments, 25(1), 62-71.

https://doi.org/10.1080/10494820.2015.1114498

Hefetz, A., \& Liberman, G. (2017). The factor analysis procedure for exploration : a short guide with examples. Cultura $y$ Educación, 29(3), 526-562. https://doi.org/10.1080/11356405.2017.1365425

Huss, J. A., Sela, O., \& Eastep, S. (2015). A Case Study of Online Instructors and Their Quest for Greater Interactivity in Their Courses: Overcoming the Distance in Distance Education. Australian Journal of Teacher Education, 40(4). https://bit.ly/2opMhc2

Jiménez-Palmero, D., Mora-Núñez, M. \& Cuadros-Muñoz. R. (2016). La importancia de las nuevas tecnologías en el proceso educativo. Propuesta didáctica TIC para ELE: "melendien7dias". Revista Fuentes, 18(2), $209-223$. http://dx.doi.org/10.12795/revistafuentes.2016.18.2.07

Kahveci, M. (2007). An instrument development: interactivity survey (the IS). In Society for Information Technology \& Teacher Education International Conference (809-819). https://bit.ly/2pQe85z

Kishabale, B. (2019). Modeling e-learning interactivity, learner satisfaction and continuance learning Intention in Ugandan higher learning institutions. International Journal of Education and Development using Information and Communication Technology (IJEDICT), 15(1), 14-34.https://bit.ly/2xrZ2XC

Kaiser, H. F. (1974). An index of factorial simplicity. Psychometrika, 39(1), 31-36.

Lloret-segura, S., Ferreres-traver, A., Hernández-Baeza, A., \& Tomás-Marco, I. (2014). Exploratory Item Factor Analysis: A practical guide revised and updated. Annals of Psychology, 30(3), 1151-1169. https://doi.org/10.6018/analesps.30.3.199361 
Madland, C., \& Richards, G. (2016). Enhancing Student-Student Online Interaction: Exploring the Study Buddy Peer Review Activity. The International Review of Research in Open and Distributed Learning, 17(3). https://doi.org/10.19173/irrodl.v17i3.2179

Mauri, T., Onrubia, J., Coll, C., \& Colomina, R. (2016). La calidad de los contenidos educativos reutilizables: diseño, usabilidad y prácticas de uso. RED. Revista de Educación a Distancia, 50(8).https://doi.org/10.6018/red/50/8 Moore, M. G. (1989). Three Types of Interaction. American Journal of Distance, 3(2), 1-7. https://doi.org/10.1080/08923648909526659

Morales, P. T., \& García, J. M. S. (2015). Diseño y validación de un cuestionario para percibir el uso de la pizarra digital. Píxel-Bit. Revista de Medios y Educación, 47, 179-194. http://dx.doi.org/10.12795/pixelbit.2015.j47.12

Nolan-Grant, C. R. (2019). The Community of Inquiry framework as learning design model: a case study in postgraduate online education. Research in Learning Technology, 27(1063519), 1-15. https://doi.org/10.25304/rlt.v27.2240

O.M.Oassim-Al-shboul, Sabiote, C. R., \& Álvarez-Rodríguez, J. (2015). Profesor perceptions of distance education in virtual environments: The case of the Education Faculty of University of Al-Yarmouk ( Jordan ). Digital Education Review, 28, 145-162. https://bit.ly/31Kipoo

Ortiz, A. M. R., \& Ortiz, C. P. M. (2019). Implementación de un modelo de juego interactivo para aprender matemáticas. Praxis \& Saber, 10(22), 115-142. https://doi.org/10.19053/22160159.v10.n22.2019.7693

Park, J. Y. (2014). Student interactivity and teacher participation : an application of legitimate peripheral participation in higher education online learning environments. Technology, Pedagogy and Education, 24(3), 389-406. https://doi.org/10.1080/1475939X.2014.935743

Prieto, G., \& Delgado, A. R. (2010). Fiabilidad y validez. Papel del Psicólogo, 31(1), 67-74. https://bit.ly/2piTIYl Ruiz-Corbella, M., \& Aguilar - Feijoo, R.M. (2017). Competencias del profesor universitario: elaboración y validación de un cuestionario de autoevaluación. RIED. Revista Iberoamericana de Educación a Distancia Superior, 8 (21), 37-65. https://doi.org/10.22201/iisue.20072872e.2017.21.212

Salmon, G. (2014). Learning Innovation: A Framework for Transformation. European Journal of Open, 17(2), 219-1027. https://doi.org/10.2478/eurodl-2014-0031

Siemens, G., Gasević, D., \& Dawson, S. (2015). Preparing for the Digital University: A review of the history and current state of distance, blended, and online learning. Athabasca AB Canada: Athabasca University.

https://doi.org/10.13140/RG.2.1.3515.8483

Solórzano-García, M., \& Navío-marco, J. (2019). Developing social entrepreneurs through distance education : the value of commitment and interactivity with the learning community. International Journal of Mobile Learning and Organisation, 13(1), 30-50. https:// doi.org/10.1504/IJMLO.2019.096466

Silva, J. (2017). Un modelo pedagógico virtual centrado en las E-actividades. RED. Revista de Educación a Distancia, 53. https://doi.org/10.2478/eurodl-2014-0031

Stewart, M. K. (2019). The Community of Inquiry Survey: An Assessment Instrument for Online Writing Courses. Computers and Composition, 52, 37-52. https://doi.org/10.1016/j.compcom.2019.01.001

Stone, C., \& Springer, M. (2019). Interactivity, connectedness and Engaging and retaining students online. Australian Journal of Adult Learning, 59(2), 146. https://bit.ly/39iRh3B

Thongsawat, S., Davidson-Shivers, G., \& Chatphoomiphong, B. (2019). Designing an Interactive Student Orientation to Build Social Connectedness. Journal of Educational Multimedia and Hypermedia, 28(1), 99-112. https://bit.ly/2PijykB Vlachopoulos, D., \& Makri, A. (2019). Online communication and interaction in distance higher education: A framework study of good practice. International Review of Education, 65, 605-632. https://doi.org/10.1007/s11159-019-09792-3 Vilanova, G. E. (2016). Modelos de interacción en ambientes virtuales de aprendizaje en la educación superior. Sistemas, Cibernética E Informática, 13(1), 77-83. https://bit.ly/2JmsFNe

Wang, Z., Chen, L., \& Anderson, T. (2014). A framework for interaction and cognitive engagement in connectivist learning contexts. The International Review of Research in Open and Distributed Learning, 15(2), 1-10. https://doi.org/10.19173/irrodl.v15i2.1709

Williamson, B., Potter, J., \& Eynon, R. (2019). New research problems and agendas in learning, media and technology: the editors' wishlist. Learning, Media and Technology, 44(2), 87-91. https://doi.org/10.1080/17439884.2019.1614953 Zangara, M. A. (2017). Interacción e interactividad en el trabajo colaborativo mediado por tecnología informática. Universidad Nacional de la Plata. https://doi.org/10.35537/10915/67175

Zhu, M., Herring, S. C., \& Bonk, C. J. (2019). Exploring presence in online learning through three forms of computermediated discourse analysis. Distance Education, 1-21. https://doi.org/10.1080/01587919.2019.1600365 